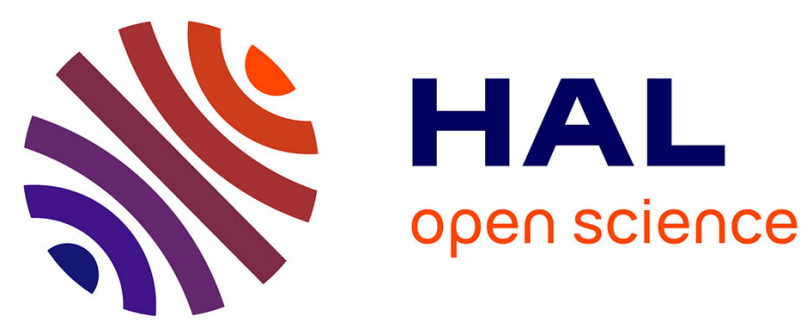

\title{
ADAPTIVE MULTITEMPORAL FILTERING OF POLARIMETRIC SAR IMAGES
}

Thu Trang Le, Abdourrahmane Atto, Emmanuel Trouvé

\section{To cite this version:}

Thu Trang Le, Abdourrahmane Atto, Emmanuel Trouvé. ADAPTIVE MULTITEMPORAL FILTERING OF POLARIMETRIC SAR IMAGES. IEEE International Geoscience and Remote Sensing Symposium (IGARSS), 2014, Jul 2014, Québec, Canada. 10.1109/IGARSS.2014.6947507 . hal01095849

\section{HAL Id: hal-01095849 \\ https://hal.science/hal-01095849}

Submitted on 16 Dec 2014

HAL is a multi-disciplinary open access archive for the deposit and dissemination of scientific research documents, whether they are published or not. The documents may come from teaching and research institutions in France or abroad, or from public or private research centers.
L'archive ouverte pluridisciplinaire HAL, est destinée au dépôt et à la diffusion de documents scientifiques de niveau recherche, publiés ou non, émanant des établissements d'enseignement et de recherche français ou étrangers, des laboratoires publics ou privés. 


\title{
ADAPTIVE MULTITEMPORAL FILTERING OF POLARIMETRIC SAR IMAGES
}

\author{
Thu Trang LÊ, Abdourrahmane M. ATTO, Emmanuel TrouvÉ \\ LISTIC, Université de Savoie, Polytech Annecy-Chambéry, \\ BP 80439 - F-74944 Annecy-le-Vieux Cedex - FrANCE
}

\begin{abstract}
This paper proposes an approach for temporal adaptive filtering of Polarimetric Synthetic Aperture Radar (PolSAR) image time series by integrating a change detection technique. The filtering strategy is based on the detection of changed and unchanged areas derived by applying an appropriate similarity test. A time series including 7 descending fine-quad polarization RADARSAT2 images acquired from January 29, 2009 to Jun 22, 2009 over the ChamonixMontBlanc test-site which includes different kinds of change is used to validate the proposed method.
\end{abstract}

Index terms - Polarimetric Synthetic Aperture Radar (PolSAR) image time series, change detection, speckle multitemporal filtering, Wishart distance, similarity test.

\section{INTRODUCTION}

Polarimetric synthetic aperture radar (PolSAR) imagery has been used extensively in many fields, such as biomass and forest height estimation, snow cover mapping, glacier monitoring, damage assessment, etc.. In particular, time series of PolSAR images obtained by repeat-pass orbits allow the temporal evolution of objects on the ground surface to be analyzed. The presence of speckle occurring in all coherent acquisition systems makes the use of PolSAR imagery difficult. Therefore, speckle filtering is often required as a preprocessing step in the exploitation process of PolSAR data.

There are many approaches in speckle reduction for PolSAR data in spatial domain, such as [1], [2], [3], [4], [5], etc.. This paper presents an adaptive filtering method for PolSAR time series based on the detection of changes in the area of interest. Different from most other multitemporal filtering methods ([6], [7], [8], etc..) which use all pixels or consecutive sub-sequences of pixels at the same spatial coordinate of the time series to estimate filtered values, the proposed method takes the presence of changes into account, only stable pixels in the pixel pile are, thus, involved in the filtering process. The changed and unchanged pixels are identified by similarity tests.

\section{FILTERING METHOD}

The scattering information of PolSAR data in monostatic and reciprocal acquisition can be addressed in a complex vector, e.g:

- Lexicographic target vector:

$$
k_{L}=\left[\begin{array}{lll}
S_{h h} & \sqrt{2} S_{h v} & S_{v v}
\end{array}\right]^{\mathcal{T}}
$$

- or Pauli target vector:

$$
k_{P}=\frac{1}{\sqrt{2}}\left[\begin{array}{lll}
S_{h h}+S_{v v} & S_{h h}-S_{v v} & 2 S_{h v}
\end{array}\right]^{\mathcal{T}}
$$

where $S_{h h}, S_{v v}$ are the scattering elements of co-polarization, $S_{h v}$ is the scattering element of cross-polarization associated with transmitted/received waves on horizontal/vertical planes and the superscript " $\mathcal{T}$ " refers to the matrix transpose.

Polarimetric information can also be presented by a covariance matrix $(C)$ based on $k_{L}$ and a coherency matrix (T) based on $k_{P}$ as follow:

$$
\begin{aligned}
& C=E\left[k_{L} \cdot k_{L}^{* \mathcal{T}}\right] \\
& T=E\left[k_{P} \cdot k_{P}^{* \mathcal{T}}\right]
\end{aligned}
$$

where the superscript "*" indicates the complex conjugate, and $E[\cdots]$ denotes mathematical expectation usually estimated by temporal or spatial averaging.

For the sake of simplicity, hereafter, we will use " $k$ " to denote $k_{L}$ or $k_{P}$ and " $T$ " to refer to $C$ or $T$.

Considering an $N$-length coregisted PolSAR image time series $\mathcal{I}=\left\{\mathcal{I}_{t}\right\}_{1 \leqslant t \leqslant N}$, where $\mathcal{I}_{t}$ denotes the image acquired at time $t$. Each pixel $\mathcal{I}_{t}(i, j)$ of image $\mathcal{I}_{t}$ is characterized by a complex matrix $T$ having the form (3) or (4). Let $\mathcal{I}_{t}^{w(i, j)}$ be the analysis window which determines the boxcar spatial neighborhood of $\mathcal{I}_{t}(i, j)$. Let $\left\{\mathcal{I}_{t}(i, j)\right\}_{1 \leqslant t \leqslant N}$ be a vector including all values of pixels at spatial position $(i, j)$, called pixel pile.

The proposed approach is made up of a processing chain including 3 steps given in Section 2.1, 2.2, and 2.3 [9]. 


\subsection{Bi-date analysis}

To determine changed and unchanged pixels in each pixel pile, similarity tests are taken between each two dates by using small spatial windows. Let $h$ denotes a similarity measure. Considering pixel spatial location $(i, j)$ observed in two different dates $t, \ell$. Let

$$
H_{t, \ell}^{w(i, j)}=h\left(\mathcal{I}_{t}^{w(i, j)}, \mathcal{I}_{\ell}^{w(i, j)}\right)_{1 \leqslant t \leqslant N, 1 \leqslant \ell \leqslant N}
$$

be the similarity degree between $\mathcal{I}_{t}(i, j)$ and $\mathcal{I}_{\ell}(i, j)$. To decide whether $H_{t, \ell}^{w(i, j)}$ represents noise only or contains some change information, it is compared to a threshold $\lambda$, therefore the similarity test is:

$$
H_{t, \ell}^{w(i, j)} \underset{1}{\stackrel{5}{\lessgtr}} \lambda .
$$

The change information is defined:

$$
M_{t, \ell}^{w(i, j)}= \begin{cases}0 & \text { if } \mathcal{I}_{\ell}^{w(i, j)} \in \Psi\left(\mathcal{I}_{t}^{w(i, j)}\right) \\ 1 & \text { if } \mathcal{I}_{\ell}^{w(i, j)} \notin \Psi\left(\mathcal{I}_{t}^{w(i, j)}\right)\end{cases}
$$

where $\Psi\left(\mathcal{I}_{t}^{w(i, j)}\right)$ is the class of pixel $\mathcal{I}_{t}(i, j)$ including pixels having positive responses to the similarity test.

Matrix $M$ is called Step 1 Change Detection Matrix (CDM1). This is a symmetric matrix with the size of $N \times$ $N$ containing 0 and 1 values, in which 0 represents "unchanged" and 1 represents "changed" pixel.

\subsection{Multidate analysis}

In order to refine the result of changed and unchanged pixel determination in each pixel pile derived from step 1 (bi-date analysis), we reconsider the similarity test given above (bidate analysis) in a multidate framework. All unchanged pixels determined in CDM1 corresponding to each two reference pixels are retested.

$$
\begin{gathered}
\hat{H}_{t, \ell}^{w(i, j)}=h\left(\Psi\left(\mathcal{I}_{t}^{w(i, j)}\right), \Psi\left(\mathcal{I}_{\ell}^{w(i, j)}\right)\right)_{1 \leqslant t \leqslant N, 1 \leqslant \ell \leqslant N} \\
\hat{M}_{t, \ell}^{w(i, j)}=\left[\hat{H}_{t, \ell}^{w(i, j)} \underset{1}{\lessgtr} \lambda\right]
\end{gathered}
$$

Matrix $\hat{M}$ is called Step 2 Change Detection Matrix (CDM2).

\subsection{Multitemporal filtering}

After step 2, CDM2s provide reliable temporal information on changed and unchanged pixels in each pile. The CDMbased estimator proposed hereafter aggregates the unchanged pixels by using the mean statistics to obtain filtered values:

$$
\hat{\mathcal{I}}_{t}(i, j)=\frac{1}{N_{\Psi_{t}(i, j)}} \sum_{\ell=1}^{N_{\Psi_{t}(i, j)}}\left(1-\hat{M}_{t, \ell}^{w(i, j)}\right) \mathcal{I}_{\ell}^{(i, j)}
$$

where, $N_{\Psi_{t}(i, j)}$ is the number of elements in class $\Psi\left(\mathcal{I}_{t}^{w(i, j)}\right)$.

\section{APPLICATION}

\subsection{Similarity test}

For implementing the proposed approach, it is crucial to determine changed and unchanged areas in the time series. This depends on the selection of an appropriate similarity measure and threshold.

\subsubsection{Test criterion}

In this paper, we have chosen the Wishart distance to measure the similarity between pixels of each PolSAR pixel pile. For the speckle reduction purpose, $n$-look image is formed by multilook processing which can be carried out by averaging $n$ independent samples of: 1) $n$ single-look images or 2) $n$ spatial neighboring single-look processed pixels.

$$
[T]=\frac{1}{n} \sum_{i=1}^{n} k_{i} k_{i}^{* \mathcal{T}}
$$

The averaged covariance/coherency matrix $[T]$ has a complex Wishart distribution [10]. The Wishart distance derived from maximum likelihood procedure on Wishart distribution assumption is:

$D_{\text {wishart }}\left([T]_{1},[T]_{2}\right)=\ln \frac{\operatorname{det}\left\{[T]_{2}\right\}}{\operatorname{det}\left\{[T]_{1}\right\}}+\operatorname{Tr}\left\{[T]_{2}^{-1}[T]_{1}\right\}$.

Thus, the similarity measure $h$ is $D_{\text {wishart }}$.

\subsubsection{Threshold selection}

To identify changed pixels, a threshold is empirically determined from training samples of test site specific content. This content relates to the different pixel classes which can be observed in the test site.

Assuming that the study area has $m$ classes, we choose the same number of training samples for each class. Then Wishart distances are calculated between pixels within each class (intraclass-distance) and between pixels of two different classes (interclass-distance) using an arbitrary image in the time series. We will have $m$ histograms of intraclassdistances and $m(m-1) / 2$ histograms of interclass-distances.

The threshold is selected as the smallest Wishart distance value at the intersection point between histogram of intraclass-distances and histogram of interclass-distances.

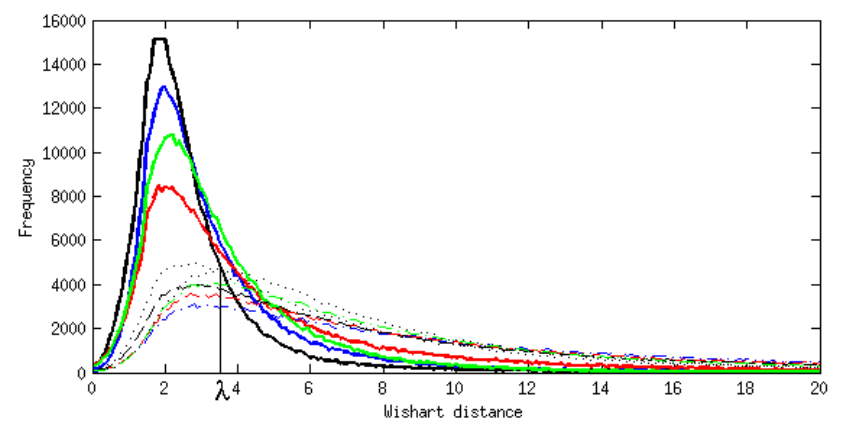

Figure 1: Histograms of Wishart distances of 4 classes. Solid lines: intraclass-distances; Dashed lines: interclassdistances 


\subsection{Experimental results}

The proposed method is illustrated on a time series including 7 descending fine-quad polarization RADARSAT2 images acquired from January 29, 2009 to Jun 22, 2009 over the Chamonix-MontBlanc test-site which includes different kinds of change.

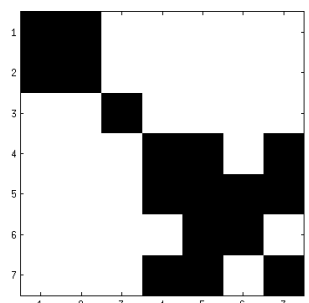

(a)

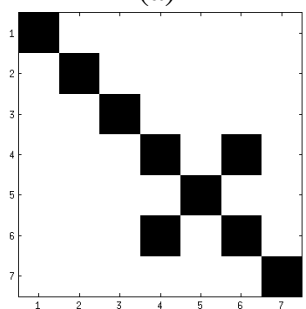

(c)

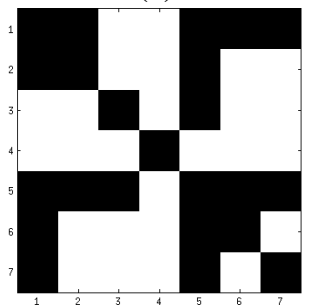

(e)

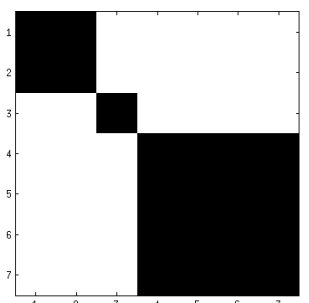

(b)

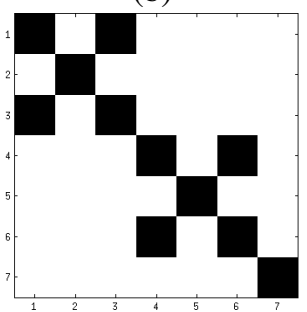

(d)

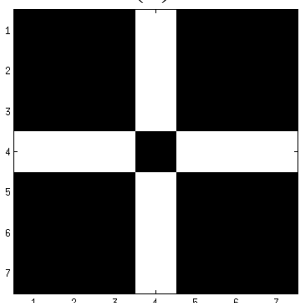

(f)
Figure 2: Change detection matrices at different areas. (a), (b): CDM1 and CDM2 at a seasonally stable area; (c), (d): CDM1 and CDM2 at a moving area; (e), (f): CDM1 and $\mathrm{CDM} 2$ at an isolated target.

Figure 2 shows CDMs at different characteristic areas which can describe properties of CDM-based filter. Indeed, looking into CDM1s (Figure 2(a), (c), (e)) and CDM2s (Figure 2(b), (d), (f)), we can observe that the proposed filtering operator detects changed/unchanged pixels in each pixel pile effectively and CDM2 is a refined detection as reducing the "false alarms" involved in CDM1. At a seasonally stable area (in Figure 2(a) and (b)), stationary periods of the time series are determined, as the black block from April 11, 2009 to Jun 22, 2009 for instance. At a moving area like glacier, CDM1 and CDM2 in Figure 2(c) and (d) show that there are a few detected unchanged pixels for the averaging process as glacier pixel values in the pile change from date to date. Hence, the CDM-based filter has a little impact on this area in order to save temporal characteristics. At an isolated target, where the pixel is definitely different from all other pixels of the pile (Figure 2(e) and (f)), the filtered value of this pixel equals the original value as preserving change information.

Filtered images in Figure 3 illustrates that the proposed method reduce speckle significantly while preserving detail features of original images. A visual comparison with IDAN filter (Figure 3 (c)) and refined LEE filter (Figure 3 (d)) shows that these filters have blurring effect on filtered images, whereas CDM-based filtered image (Figure 3 (b)) presents obviously textures with small details which cannot be distinguished in the original image.

\section{CONCLUSIONS}

The proposed filter operates a nonlocal temporal selection on unchanged pixels and aggregates those unchanged pixels by using an unbiased estimator of the mean value. There is no spatial and temporal degradation in the filtered images because the estimator uses only the selected temporal information (pertaining to the stable class) in the averaging process.

The experimental results have shown that the proposed approach reduces speckle significantly while preserving detail features: edges (spatial image characteristics) and changes (temporal evolution information). The filter has very little impact on moving areas. This advantage allows images including stable and moving areas to be filtered without losing characteristics of moving areas which are very important in monitoring their evolution (glacier for instance).

\section{REFERENCES}

[1] J.S. Lee, M.R.Grunes, and S.A. Mango, "Speckle reduction in multipolarization and multifrequency SAR imagery," IEEE Trans. Geosci. Remote Sens., vol. 29, no. 4, pp. 535-544, 1991.

[2] A. Lopes and F. Sery, "Optimal speckle reduction for the product model in multilook polarimetric sar imagery and the whishart distribution," IEEE Trans. Geosci. Remote Sens., vol. 35, no. 3, pp. 632-647, 1997.

[3] G. Vasile, E. Trouve, J.-S. Lee, and V. Buzuloiu, "Intensity-driven adaptive-neighborhood technique for polarimetric and interferometric SAR parameters estimation," IEEE Trans. Geosci. Remote Sens., vol. 44, no. 6, pp. 1609-1621, 2006.

[4] J.S.Lee, M.R. Grunes, D.L.Schuler, E.Pottier, and L.Ferro-Famil, "Scattering-model-based speckle filtering of polarimetric SAR data," IEEE Trans. Geosci. Remote Sens., vol. 44, no. 1, pp. 176-187d, 2006. 

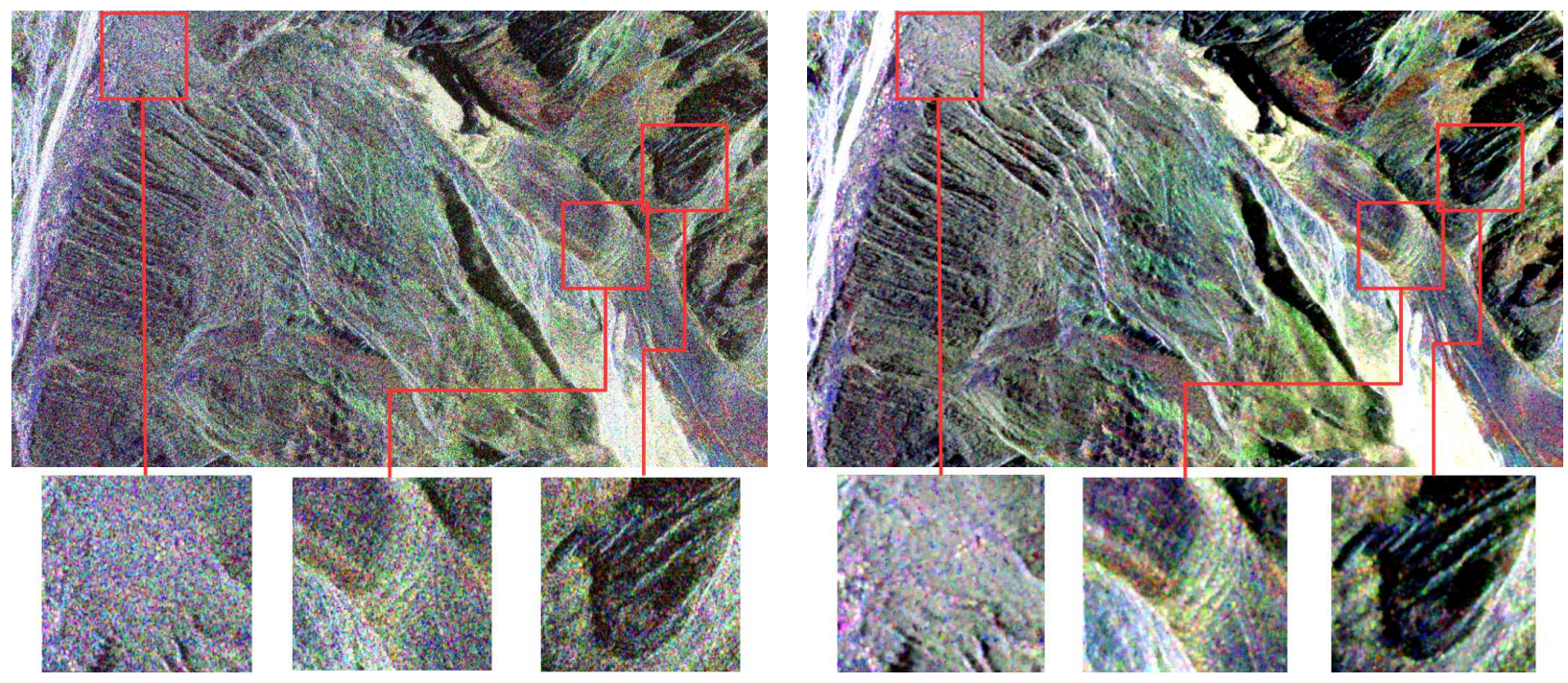

(a) Original image

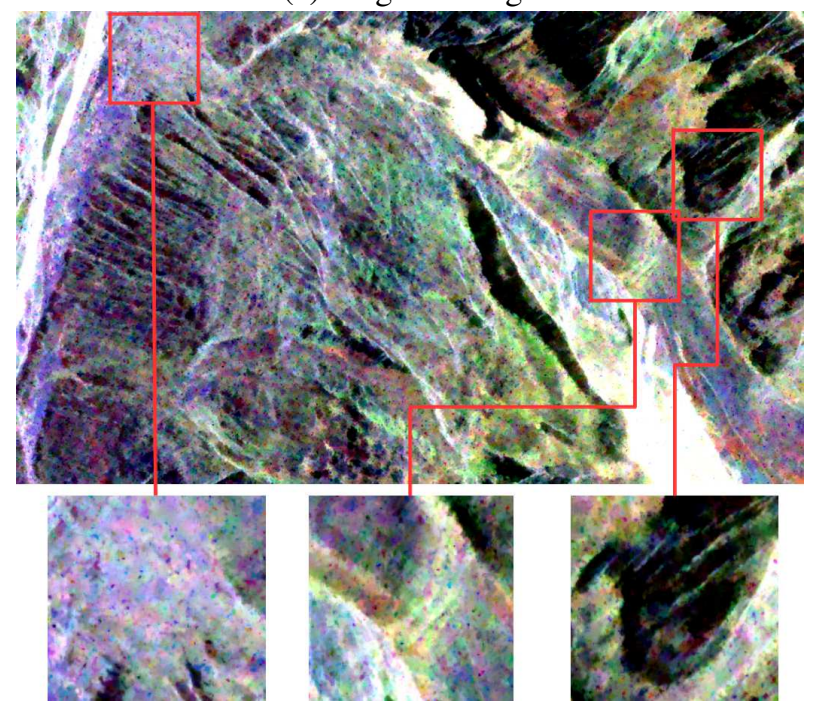

(c) IDAN filtered image

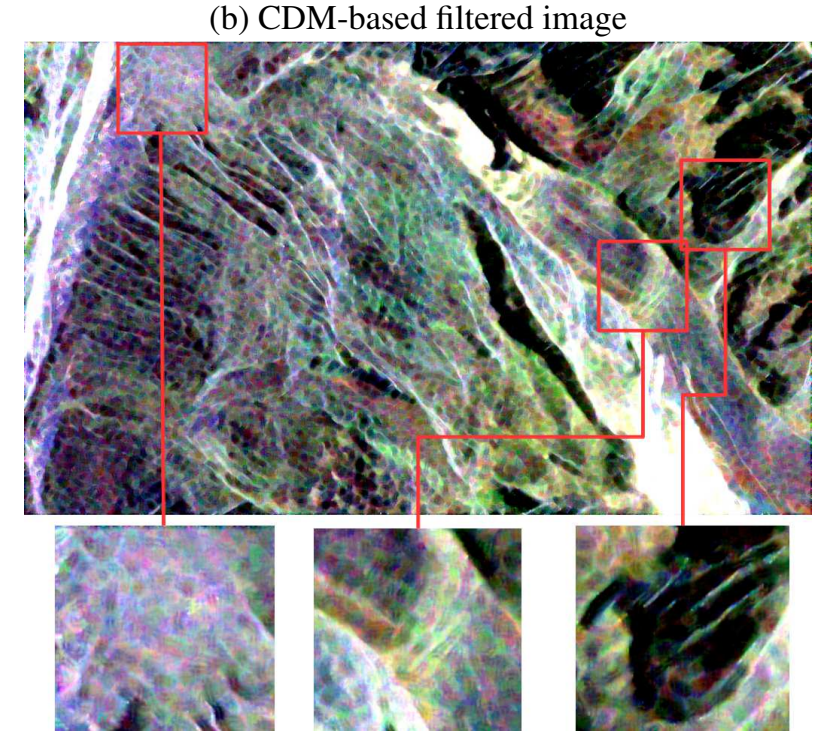

(d) Refined LEE filtered image

Figure 3: Filtering result of image acquired on January 29, 2009 in Pauli basis

[5] A. Alonso-Gonzalez, C. Lopez Martinez, and P. Salenbier, "Filtering and segmentation of polarimetric SAR images with binary partition tree," in Proc. IGARSS Honolulu, USA, pp. 4043-4046, July 2010.

[6] J. Bruniquel and A. Lopes, "Multi-variate optimal speckle reduction in SAR imagery," Int. J. Remote Sens., vol. 18, no. 3, pp. 603-627, 1997.

[7] D. Coltuc, E. Trouve, F. Bujor, N. Classeau, and J. P. Rudant, "Time-space filtering of multitemporal SAR images," in Proc. IGARSS Honolulu, USA, vol. 7, pp. 2909-2911, 2000.

[8] M. Ciuc, Ph. Bolon, E. Trouve, V. Buzuloiu, and J-P.
Rudant, "Adaptive-neighborhood speckle removal in multitemporal SAR images," Appl. Opt., vol. 40, no. 32, pp. 5954-5966, 2001.

[9] T. T. Le, A. M. Atto, E. Trouve, and J. M. Nicolas, "Adaptive multitemporal SAR image filtering based on the change detection matrix," IEEE Geosci. Remote Sens. Lett., vol. 11, no. 10, pp. 1826-1830, 2014.

[10] J.S. Lee, M.R. Grunes, T.L. Ainsworth, L.J. Du, D.L. Schuler, and S.R. Cloude, "Unsupervised classification using polarimetric decomposition and the complex wishart classifier," IEEE Trans. Geosci. Remote Sens., vol. 37, no. 5, pp. 2249-2258, 1999. 